\title{
THE STRUCTURE OF CROSSED PRODUCTS BY SMOOTH ACTIONS
}

\author{
DANA P. WILLIAMS
}

(Received 13 November 1987)

Communicated by I. Raeburn

\begin{abstract}
Let $\xi$ be a $C^{*}$-bundle over $T$ with fibres $\left\{A_{t}\right\}_{l \in A}$. Suppose that $A$ is the $C^{*}$-algebra of sections of $\xi$ which vanish at infinity, and that $(A, G, \alpha)$ is a $C^{*}$-dymanical system such that, for each $t \in T$, the ideal $I_{t}=\{f \in A \mid f(t)=0\}$ is $G$-invariant. If in addition, the stabiliser group of each $P \in \operatorname{Prim}(A)$ is amenable, then $A \rtimes_{a} G$ is the section algebra of a $C^{*}$-bundle with fibres $\left\{A_{t} \rtimes_{\alpha} G\right\}_{t \in T}$.

The above theorem may be used to prove a structure theorem for crossed products built from $C^{*}$-dynamical systems $(A, G, \alpha)$ where the action of $G$ on $A$ is smooth. Assuming that the stabiliser groups are amenable, then $A \rtimes_{\alpha} G$ has a composition series such that each quotient is a section algebra of a $C^{*}$-bundle where the fibres are of the form $A_{\delta} \rtimes_{\alpha} G$; moreover, the $A_{\delta}$ correspond to locally closed subsets of $\operatorname{Prim}(A)$, and $G$ acts transitively on $\operatorname{Prim}\left(A_{\delta}\right)$. In many cases, in particular when $(G, A)$ is separable, the $A_{\delta} \rtimes_{\alpha} G$ have been computed explicitly by other authors.
\end{abstract}

These results are actually proved for twisted $C^{*}$-dynamical systems.

1980 Mathematics subject classification (Amer. Math. Soc.) (1985 Revision): 46 L 05.

\section{Introduction}

It is a classical theorem in the theory of $C^{*}$-algebras that type I $C^{*}$-algebras have composition series of ideals $\left\{J_{\delta} \mid 0 \leq \delta \leq \delta_{0}\right\}$, such that the quotients, $J_{\delta+1} / J_{\delta}$, have tractable properties. For instance one may insist that the quotient algebras be GCR, CCR, or even have continuous trace ([3], [14]). In this paper, it will be shown that, under suitable hypotheses, crossed products, $A \rtimes_{\alpha} G$, for which the action of $G$ on $A$ is smooth, have composition series

(C) 1989 Australian Mathematical Society 0263-6115/89 \$A2.00+0.00 
in which the quotients are section algebras of $C^{*}$-bundles with fibres that are smaller and hopefully, more manageable than the original algebra (Theorem 3.2). As a corollary, one can prove, with slightly different hypotheses, a theorem of Takesaki characterizing type I crossed product algebras when the action is smooth [16, Theorem 6.1].

To prove the above, a theorem about crossed products of section algebras is required. Namely, suppose that $(A, G, \alpha)$ is an $E H$-regular $C^{*}$-dynamical system with each stabiliser group for the action of $G$ on Prim $A$ amenable, and that $A=\Gamma_{0}(\xi)$, where $\xi$ is a $C^{*}$-algebra bundle over $T$ with fibres $\left\{A_{t} \mid t \in T\right\}$. If the kernel of the natural map of $A$ onto $A_{t}$ is $G$-invariant for every $t \in T$, then $A \rtimes_{\alpha} G$ is the $C^{*}$-algebra of sections of a $C^{*}$-bundle over $T$ with fibres $\left\{A_{t} \rtimes_{\alpha} G \mid t \in T\right\}$ (Theorem 2.1). For example, suppose that $A \cong C_{0}(\Omega)$, so that $(G, \Omega)$ is a locally compact transformation group, and that $(G, \Omega)$ is second countable. If $\Omega / G$ is Hausdorff, then $A \rtimes_{\alpha} G$ is a section algebra over $\Omega / G$ and the fibre over $G \cdot x$ is isomorphic to $C^{*}\left(G_{x}\right) \otimes \mathscr{K}\left(L^{2}\left(G / G_{x}, \mu\right)\right)$, where $G_{x}$ is the stabiliser group at $x$ and $\mu$ is any quasi-invariant measure on $G / G_{x}$ (cf. Corollary 3.3).

Unless stated otherwise, all ideals are assumed to be closed and two-sided. Homomorphisms between $C^{*}$-algebras will always be *-preserving and representation will mean non-degenerate *-representation. If $A$ and $B$ are subalgebras of $C$, then $A \cdot B$ denotes the closed linear span of products from $A$ and $B$. If $A$ is a $C^{*}$-algebra, then $\mathscr{M}(A)$ denotes the multiplier algebra of $A$ ([1]), and $\mathscr{T}(A)$ denotes the space of ideals of $A$ endowed with the inner hull-kernel topology (cf. [8, page 208], or the remarks preceding Lemma 2.4 in [17]). Recall that a sub-basis for the topology on $\mathscr{T}(A)$ is

$$
\left\{\mathscr{O}_{J}\right\}_{J \in \mathscr{T}(A)} \text {, where } \mathscr{O}_{J}=\{I \in \mathscr{T}(A) \mid I \nsupseteq J\} .
$$

The majority of the research for this paper was carried out while the author was a student at the University of California at Berkeley. The results, in slightly weaker form, formed part of the author's doctoral dissertation written under the supervision of Marc Rieffel. Also, at the suggestion of others, the author reorganized the proof of Theorem 2.1 so that [13, Theorem 4] could be used directly.

I would like to take the opportunity to thank Professor Rieffel for his help and guidance during the writing of this dissertation.

\section{Crossed products of section algebras}

For the basic facts concerning crossed product algebras the reader is encouraged to consult [2], [4], [14], and [16]. The standard reference for twisted 
crossed products is [8], where they were studied under the name of twisted covariance algebras. For convenience, we establish some notation and state some essential facts. Let $(A, G, \alpha)$ be a dynamical system. The crossed product algebra, $A \rtimes_{\alpha} G$, is the enveloping $C^{*}$-algebra of $L^{1}(G, A)$, where $L^{1}(G, A)$ is the Banach $*$-algebra of all Bochner integrable $A$-valued functions on $G$ with respect to a fixed left Haar measure on $G$. Multiplication and involution are defined by

$$
\begin{gathered}
f * g(s)=\int_{G} f(r) \alpha_{r}\left(g\left(r^{-1} s\right)\right) d r, \\
f^{*}(s)=\Delta\left(s^{-1}\right) \alpha_{s}\left(f\left(s^{-1}\right)\right)^{*} .
\end{gathered}
$$

A covariant representation, $\left(V_{L}, M_{L}\right)$, of $(G, A)$ gives a non-degenerate representation, $L$ of $A \rtimes_{\alpha} G$ whose action on $f \in C_{c}(G, A)$ is given by

$$
L(f)=\int_{G} M_{L}(f(s)) V_{L}(s) d s .
$$

Note that the representation of $A \rtimes_{\alpha} G$ on itself by left multiplication is the integrated form of $(V, M)$ where $V(s) f(r)=\alpha_{s}\left(f\left(s^{-1} r\right)\right)$ and $M(a) f(r)=a f(r)$ for $s, r \in G, a \in A$, and $f \in C_{c}(G, A)$. These actions induce homomorphisms $R_{G}$ and $R_{A}$ of $G$ and $A$ into $\mathscr{M}\left(A \rtimes_{\alpha} G\right)$ [8].

A twisted $C^{*}$-dynamical system $(A, G, \alpha, \tau)$ is a dynamical system $(A, G, \alpha)$ together with a continuous homomorphism $\tau$ of a closed normal subgroup $N_{\tau}$ of $G$ into the group of unitaries in $\mathscr{M}(A)$, equipped with the strict topology, and such that

$$
\tau(n) a \tau\left(n^{-1}\right)=\alpha_{n}(a),
$$

and

$$
\tau\left(s n s^{-1}\right)=\tilde{\alpha}_{s}(\tau(n)),
$$

where $n \in N_{\tau}, s \in G, a \in A$, and $\tilde{\alpha}_{s}$ is the canonical extension of $\alpha_{s}$ to $\mathscr{M}(A)$. If $I$ is the ideal of $\mathscr{K}\left(A \rtimes_{\alpha} G\right)$ generated by $\left\{R_{G}(n) R_{A}(a)-\tau(n) R_{A}(a) \mid n \in N_{\tau}\right.$ and $a \in A\}$, then the twisted crossed product $A \rtimes_{\alpha}^{\tau} G$ is defined to be the quotient $A \rtimes_{\alpha} G /\left(I \cap A \rtimes_{\alpha} G\right)$. Notice that the representations of $A \rtimes_{\alpha}^{\tau} G$ are exactly those corresponding to covariant pairs $\left(V_{L}, M_{L}\right)$ which satisfy $V_{L}(n)=\widetilde{M}_{L}(\tau(n))$ for all $n \in N_{\tau}$. Of course, we have homomorphisms $R_{G}^{\tau}$ and $R_{A}^{\tau}$ of $G$ and $A$ in $\mathscr{M}\left(A \rtimes_{\alpha}^{\tau} G\right)$ by composing $R_{G}$ and $R_{A}$ with the quotient map.

Since it will be convenient to make considerable use of the maps between spaces of ideals introduced in [8], we include the basic definitions.

Firstly, there is a map Ex from $\mathscr{T}(A)$ to $\mathscr{T}\left(A \rtimes_{\alpha}^{\tau} G\right)$ defined by

$$
\operatorname{Ex}(I)=\text { ideal generated by }\left\{R_{A}^{\tau}(a) f \mid a \in I, f \in A \rtimes_{\alpha}^{\tau} G\right\} .
$$


(See [8, Proposition 9].) If in addition, $I$ is $G$-invariant, then $\operatorname{Ex}(I)$ can be identified with $I \rtimes_{\alpha}^{\tau} G$ [8, Proposition 12]. Here we have engaged in a slight abuse of notation and have used $\alpha$ to denote the obvious action of $G$ on $I$, and $\tau$ to denote the twist on $(I, G, \alpha)$ obtained by composing $\tau$ with the natural map of $\mathscr{M}(A)$ into $\mathscr{M}(I)$. Similarly, we will write $(A / I) \rtimes_{\alpha}^{\tau} G$ for the crossed product of $A / I$ by the action of $G$ obtained from $\alpha$ with the twist arising from the composition of $\tau$ with the natural map of $\mathscr{M}(A)$ into $\mathscr{M}(A / I)$.

Secondly, if $N_{\tau} \subseteq H$, we let $E_{H}^{G}=C_{0}(G / H, A) \rtimes_{\alpha}^{\tau} G$ be the imprimitivity algebra defined in [8, Section 2]; the action of $G$ on $C_{0}(G / H, A)$ is the diagonal action, $\tilde{\alpha}_{s}(\phi)(r H)=\alpha_{s}\left(\phi\left(s^{-1} r H\right)\right)$. Moreover, the natural representation of $A \rtimes_{\alpha}^{\tau} G$ on $E_{H}^{G}$ defines a homomorphism, $L^{H}$, of $A \rtimes_{\alpha}^{\tau} G$ into $\mathscr{M}\left(E_{H}^{G}\right)$. In fact, the representation of $A \rtimes_{\alpha}^{\tau} G$ induced from $\pi$ on $A \rtimes_{\alpha}^{\tau} H$ is obtained by first inducting $\pi$ to $E_{H}^{G}$, then extending to $\mathscr{M}\left(E_{H}^{G}\right)$, and finally restricting to the image of $A \rtimes_{\alpha}^{\dagger} G$. Then we can define a continuous map, $\operatorname{Ind}_{H}^{G}$, from $\mathscr{T}\left(A \rtimes_{\alpha}^{\tau} H\right)$ to $\mathscr{T}\left(A \rtimes_{\alpha}^{\tau} G\right)$ by

$$
\operatorname{Ind}_{H}^{G}(I)=\left\{f \in A \rtimes_{\alpha}^{\tau} G \mid L^{H}(f) \cdot E_{H}^{G} \subseteq(I)^{E_{H}^{G}}\right\},
$$

where $(I)^{E_{H}^{G}}$ is the ideal of $E_{H}^{G}$ corresponding to $I$ by [15, Theorem 3.1].

Recall that $(G, \operatorname{Prim}(A))$ is a topological transformation group with the $G$-action given by $s \cdot P=\left\{\alpha_{s}(a) \mid a \in P\right\}$ [10, Lemma 1.3]. We will want to look at crossed products which are $E H$-regular. For our purposes $A \rtimes_{\alpha}^{\tau} G$ is $E H$-regular if every primitive ideal of $A \rtimes_{\alpha}^{\tau} G$ is of the form $\operatorname{Ind}_{G_{P}}^{G}(I)$, where $I$ is a primitive ideal of $A \rtimes_{\alpha}^{\tau} G_{P}$ and $G_{P}$ is a stabiliser group for the action of $G$ on $\operatorname{Prim}(A)$. For the complete definition of $E H$-regularity, the reader should consult [8].

Let $\xi$ be a $C^{*}$-bundle over a locally compact Hausdorff space $T$ with fibre algebras $\left\{A_{t}\right\}_{t \in T}$ (see [5, Section 1] or [7, Section 1] for the definition a $C^{*}$ bundle). Those $C^{*}$-algebras which arise as the section algebra $A=\Gamma_{0}(\xi)$ of a $C^{*}$-bundle $\xi$ have recieved considerable attention over the years. For example, such algebras have been studied under the name of "a maximal full algebra of operator fields" in [13] and [6], and "the $C^{*}$-algebra defined by a continuous field of $C^{*}$-algebras" in [3]. Fortunately, it follows from [7, Proposition 1.6], that all these notions coincide. In particular, $A$ may be realized as the section algebra of a $C^{*}$-bundle $\xi$ over $T$ if and only if there is a continuous open surjection of $\operatorname{Prim}(A)$ onto $T$ [13, Theorem 4].

The rest of this section will be devoted to proving

THEOREM 2.1. Suppose that $\xi$ is a $C^{*}$-bundle over a locally compact Hausdorff space $T$ with fibres $\left\{A_{t}\right\}_{t \in T}$, and that $A=\Gamma_{0}(\xi)$. If $(A, G, \alpha, \tau)$ is a twisted $C^{*}$-dynamical system such that (1) $A \rtimes_{\alpha}^{\tau} G$ is EH-regular, (2) each ideal $I_{t}=\{a \in A \mid a(t)=0\}$ is G-invariant, and (3) for each $P \in \operatorname{Prim}(A)$, the 
group $G_{P} / N_{\tau}$ is amenable, then $A \rtimes_{\alpha}^{\tau} G$ is the section algebra of a $C^{*}$-bundle over $T$ with fibres $\left\{A_{t} \rtimes_{\alpha}^{\tau} G\right\}_{t \in T}$.

Before proceeding with the proof of the theorem, a few remarks are in order. As the statement of the theorem appears to be obvious, it is surprising that the proof is not immediate. One easily obtains a Banach bundle over $T$ whose fibres are quotients of $L^{1}(G, A)$, but it is unclear what happens when passing to the $C^{*}$-completion.

The approach here uses the characterization of section algebras over $T$ in terms of the topology on the primitive ideal space due to $R$. $Y$. Lee mentioned above. Notice that, if $G$ is amenable, then $A \rtimes_{\alpha}^{\tau} G$ is $E H$-regular by [12]. In fact, when $G$ is amenable the proof of the theorem follows almost immediately from [8, Proposition 13(ii)]. However, the added generality in the statement of the theorem will be useful in the next section.

Notice that the natural action of $C_{0}(T)$ on $A$ allows us to define homomorphisms, $R_{T}$ of $C_{0}(T)$ into $\mathscr{M}\left(A \rtimes_{\alpha}^{\tau} G\right)$, and $R_{T}^{H}$ into $\mathscr{M}\left(E_{H}^{G}\right)$. In fact, it is not difficult to check that the resulting actions of $C_{0}(T)$ are non-degenerate on $A \times_{\alpha}^{\tau} G$ and $E_{H}^{G}$. Moreover, since the $I_{t}$ are $G$-invariant, both $R_{T}\left(C_{0}(T)\right)$ and $R_{T}^{H}\left(C_{0}(T)\right)$ are central. The next lemma is an immediate consequence of these remarks.

LEMMA 2.2. If $L$ and $M$ are irreducible representations of $A \rtimes_{\alpha}^{\tau} G$ and $E_{H}^{G}$ respectively, then there are $t$ and $s$ in $T$ such that $L\left(R_{T}(\phi) f\right)=\phi(t) L(f)$ and $M\left(R_{T}^{H}(\phi) g\right)=\phi(s) M(g)$ for all $\phi \in C_{0}(T), f \in A \rtimes_{\alpha}^{\tau} G$, and $g \in E_{H}^{G}$.

The next lemma is the essential fact needed for the proof of the theorem; it is the only place amenability or $E H$-regularity is required.

LEMMA 2.3. In the same situation as Theorem 2.1, if $F$ is any subset of $T$, then

$$
\left(\bigcap_{t \in F} I_{t}\right) \rtimes_{\alpha}^{\tau} G=\bigcap_{t \in F}\left(I_{t} \rtimes_{\alpha}^{\tau} G\right) .
$$

Proof. We clearly have $\left(\bigcap_{F} I_{t}\right) \rtimes_{\alpha}^{\tau} G \subseteq \bigcap_{F}\left(I_{t} \rtimes_{\alpha}^{\tau} G\right)$. If the lemma were false, there would be a $g$ contained in the right-hand side, but not in the left. By $E H$-regularity, there is a primitive ideal of the form $P=\operatorname{Ker}(L)$, and $L=\operatorname{Ind}_{H}^{G}(\pi)$ with $H / N_{\tau}$ amenable such that $P$ contains $\left(\bigcap_{F} I_{t}\right) \rtimes_{\alpha}^{\tau} G$ and $g \notin P$.

Let $t \in T$ be the point associated to $L$ by Lemma 2.4. Clearly, $t \in \bar{F}$. We will make use of the following fact: if $C$ is a closed subset of $T$ and $J_{C}$ the ideal of functions in $C_{0}(T)$ vanishing on $C$, then $\left(\bigcap_{C} I_{t}\right) \rtimes_{\alpha}^{\tau} G=\operatorname{Ex}\left(\bigcap_{C} I_{t}\right)=$ 
$\operatorname{Ex}\left(J_{C} \cdot A\right)=R_{T}\left(J_{C}\right) \cdot A \rtimes_{\alpha}^{\tau} G$. Applying this observation with $C=\{t\}$ and using [15, Theorem 3.1], it follows that $\left(I_{t} \rtimes_{\alpha}^{\tau} H\right)^{E_{H}^{G}}=R_{T}^{H}\left(J_{t}\right) \cdot E_{H}^{G}$. In particular, since $L^{H}\left(R_{T}(\phi) f\right)=R_{T}^{H}(\phi) L^{H}(f), g \in \operatorname{Ind}_{H}^{G}\left(I_{t} \rtimes_{\alpha}^{\tau} H\right)=\{f \in$ $\left.A \rtimes_{\alpha}^{\tau} G \mid L^{H}(f) \cdot E_{H}^{G} \subseteq R_{T}^{H}\left(J_{t}\right) \cdot E_{H}^{G}\right\}$, for each $t \in F$. Since $H / N_{\tau}$ is amenable, $I_{t} \rtimes_{\alpha}^{\tau} H=\operatorname{Ex}_{N_{\tau}}^{H}\left(I_{t}\right)=\operatorname{Ind}_{N_{\tau}}^{H}\left(I_{t}\right)$ by [8, Proposition 13(ii)]. Since $\operatorname{Ind}_{H}^{G}$ and $\operatorname{Ind}_{N_{\tau}}^{H}$ are intersection preserving [8, Proposition 9], $g \in \operatorname{Ind}_{H}^{G}\left(\left(\cap_{F} I_{t}\right) \rtimes_{\alpha}^{\tau} H\right)$. In short, $L^{H}(g) E_{H}^{G} \subseteq R_{T}^{H}\left(J_{F}\right) \cdot E_{H}^{G}$, where $J_{F}$ is the ideal of functions in $C_{0}(T)$ vanishing on $F$ (hence $\bar{F}$ ). Since $t \in \bar{F}$, it follows that $L(g)=0$, a contradiction.

Proof of Theorem 2.1. Lemma 2.2 allows us to associate a unique element of $T$ with each primitive ideal of $A \rtimes_{\alpha}^{\tau} G$; the resulting map from Prim $A \rtimes_{\alpha}^{\tau} G$ onto $T$ will be denoted by $\beta$. It will suffice in view of [13, Theorem 4], to show that $\beta$ is continuous and open. In that case, $A \rtimes_{\alpha}^{\tau} G$ is a section algebra over $T$ with fibre algebras $A \rtimes_{\alpha}^{\tau} G / I_{t} \rtimes_{\alpha}^{\tau} G$. By [8, Proposition 8], these fibres are isomorphic to $A_{t} \rtimes_{\alpha}^{\tau} G$.

Note that $\beta$ is essentially the restriction of the map $R_{T}^{*}: \mathscr{T}\left(A \rtimes_{\alpha}^{\tau} G\right) \rightarrow$ $\mathscr{T}\left(C_{0}(T)\right)$ to $\operatorname{Prim}\left(A \times_{\alpha}^{\tau} G\right)$, where $R_{T}^{*}$ is defined by

$$
R_{T}^{*}(I)=\left\{\phi \in C_{0}(T) \mid R_{T}(\phi) A \rtimes_{\alpha}^{\tau} G \subseteq I\right\} .
$$

Thus, $\beta$ is continuous [8, Proposition 9].

Now suppose that $\mathscr{O}$ is open in Prim $A \rtimes_{\alpha}^{\tau} G$, that $t_{n} \notin \beta(\mathscr{O})$, and that $t_{n} \rightarrow t$. It follows from Lemma 2.4 that $I_{t_{n}} \rtimes_{\alpha}^{\tau} G$ converges to $I_{l} \rtimes_{\alpha}^{\tau} G$. This implies that no primitive ideal in $\beta^{-1}(t)$ lies in $\mathscr{O}$. This follows since whenever $I_{t} \rtimes_{\alpha}^{\tau} G \nsupseteq J$, for some ideal $J$ in $A \rtimes_{\alpha}^{\tau} G$, the same must eventually be true for $I_{t_{n}} \rtimes_{\alpha}^{\tau} G$. It follows that there is a primitive ideal, $P_{n} \supseteq I_{t_{n}} \rtimes_{\alpha}^{\tau} G$, such that $P_{n} \nsupseteq J$. Of course, $\beta\left(P_{n}\right)=t_{n}$. Thus $\beta(\mathscr{O})$ is open.

\section{The structure of smooth crossed products}

The purpose of this section is to study twisted crossed product algebras for which the action of $G$ on $A$ is rather well-behaved. In order to apply Theorem 2.1 , it will be necessary to assume the stability groups are amenable. In addition, we want the following condition to hold.

Definition 3.1. $G$ is said to act smoothly on $A$ whenever $\operatorname{Prim}(A) / G$ is almost Hausdorff (that is, every closed subset has a dense relatively open Hausdorff subset). 
This also implies that $A \rtimes_{\alpha}^{\tau} G$ is quasi-regular [8, Corollary 19]. Therefore, if the natural maps of $G / G_{P}$ onto $G \cdot P$, defined by $r G_{P} \rightarrow r \cdot P$, are homeomorphisms for each $P \in \operatorname{Prim} A$, then $(G, A)$ is regular [8, page 223], and in particular, $E H$-regular [8, Proposition 20]. In short, we may apply Theorem 2.1 to such algebras. A well-known theorem of Glimm [10], implies that if $G$ and $\operatorname{Prim}(A)$ are second countable and $\operatorname{Prim}(A)$ is also almost Hausdorff, then Definition 3.1 is equivalent to the natural maps of $G / G_{P}$ onto $G \cdot P$ being homeomorphisms for all $P$.

Recall that if $A$ is separable, then $\operatorname{Prim} A$ is second countable. If $A$ is also type I, then Prim $A$ is almost Hausdorff. However, for the moment we assume only that the $G$-action is smooth.

For convenience, we denote $\operatorname{Prim}(A) / G$ by $\Gamma$. Let $\sigma$ be the natural map of $\operatorname{Prim}(A)$ onto $\Gamma$. Recall that $\sigma$ is both continuous and open. Since $\Gamma$ is almost Hausdorff and $\operatorname{Prim}(A)$ is locally compact, the discussion on page 125 of [10] shows that there is a family of open sets, $\left\{U_{\delta}\right\}$, in $\Gamma$ indexed by ordinals $\left\{0 \leq \delta \leq \delta_{0}\right\}$ such that

(a) $\delta<\lambda$ implies $U_{\delta} \subseteq U_{\lambda}$,

(b) $U_{0}=\varnothing$ and $U_{\delta_{0}}=\Gamma$,

(c) if $\delta$ is a limit ordinal then, $U_{\delta}=\bigcup_{\lambda<\delta} U_{\lambda}$,

(d) $U_{\delta+1} \backslash U_{\delta}$ is Hausdorff in the relative topology.

With respect to the above, we make the following conventions. For $F$ closed in $\Gamma$, let $I_{F}=\bigcap_{\sigma(P) \in F} P$, a $G$-invariant ideal of $A$. Let $I_{\delta}$ be the $G$ invariant ideal of $A$ equal to $I_{U_{\delta}^{c}}$. Denote the restriction of $\sigma$ to Prim $I_{\delta}$ by $\sigma_{\delta}$. Note that $\sigma_{\delta}$ maps onto $U_{\delta}$. Let $\beta_{\delta+1}$ be the restriction of $\sigma$ to $\operatorname{Prim}\left(I_{\delta+1} / I_{\delta}\right)$, a locally closed $G$-invariant subset of $\operatorname{Prim}(A)$. Of course, $\beta_{\delta+1}$ maps onto $U_{\delta+1} \backslash U_{\delta}$.

Notice that for each $\delta$, the maps $\sigma_{\delta}$ and $\beta_{\delta+1}$ are continuous and open. Now let $F$ be closed in $U_{\delta+1} \backslash U_{\delta}$. By the continuity of $\beta_{\delta+1}$,

$$
I_{F}^{\delta+1}=\bigcap\left\{\bar{P} \in \operatorname{Prim}\left(I_{\delta+1} / I_{\delta}\right) \mid \beta_{\delta+1}(\bar{P}) \in F\right\}
$$

is a $G$-invariant ideal of $I_{\delta+1} / I_{\delta}$. In particular, since $U_{\delta+1} \backslash U_{\delta}$ is Hausdorff, $I_{\omega}^{\delta+1}$ is defined for each $\omega \in U_{\delta+1} \backslash U_{\delta}$. We let $A_{\omega}^{\delta+1}$ denote the quotient of $I_{\delta+1} / I_{\delta}$ by $I_{\omega}^{\delta+1}$. Note that $G$ acts transitively on Prim $A_{\omega}^{\delta+1}$.

THEOREM 3.2. Let $(A, G, \alpha, \tau)$ be a twisted $C^{*}$-dynamical system. Assume that $A \rtimes_{\alpha}^{\tau} G$ is $E H$-regular and that the groups, $G_{P} / N_{\tau}$, are amenable for each $P \in \operatorname{Prim}(A)$. Then with the notation described above, $\left\{I_{\delta} \rtimes_{\alpha}^{\tau} G\right\}$ forms a family of ideals in $A \rtimes_{\alpha}^{\tau} G$ such that

(1) $\delta<\lambda$ implies $I_{\delta} \rtimes_{\alpha}^{\tau} G \subseteq I_{\lambda} \rtimes_{\alpha}^{\tau} G$,

(2) $I_{0} \rtimes_{\alpha}^{\tau} G=0$ and $I_{\delta_{0}} \rtimes_{\alpha}^{\tau} G=A \rtimes_{\alpha}^{\tau} G$,

(3) If $\delta$ is a limit ordinal, then $I_{\delta} \rtimes_{\alpha}^{\tau} G$ is the ideal generated by $\left\{I_{\lambda} \rtimes_{\alpha}^{\tau} G\right\}_{\lambda<\delta}$, 
(4) $\left(I_{\delta+1} \rtimes_{\alpha}^{\tau} G\right) /\left(I_{\delta} \rtimes_{\alpha}^{\tau} G\right)$ is isomorphic to $\left(I_{\delta+1} / I_{\delta}\right) \rtimes_{\alpha}^{\tau} G$ and is the section algebra of a $C^{*}$-bundle over $U_{\delta+1} \backslash U_{\delta}$ with fibres $A_{\omega}^{\delta+1} \rtimes_{\alpha}^{\tau} G, \omega \in U_{\delta+1} \backslash U_{\delta}$.

Moreover, if $L$ is an irreducible representation of $A \rtimes_{\alpha}^{\tau} G$, then there is an $\delta<\delta_{0}$ and $a \omega \in U_{\delta+1} \backslash U_{\delta}$ such that $L$ is the canonical extension of an irreducible representation of $A_{\omega}^{\delta+1} \rtimes_{\alpha}^{\tau} G$.

Proof. Statement (1) follows from condition (a) above as Ex preserves containment. Statement (2) follows immediately from condition (b). Since Ex preserves arbitrary unions of ideals [8, Proposition 9], statement (3) follows from condition (c). We show that statement (4) follows from condition (d). First, $I_{\delta+1} \rtimes_{\alpha}^{\tau} G / I_{\delta} \rtimes_{\alpha}^{\tau} G$ is isomorphic to $\left(I_{\delta+1} / I_{\delta}\right) \rtimes_{\alpha}^{\tau} G$ by [8] Proposition 12. Since $\beta_{\delta+1}: \operatorname{Prim}\left(I_{\delta+1} / I_{\delta}\right) \rightarrow U_{\delta+1} \backslash U_{\delta}$ is continuous and open, by [13, Theorem 4], $I_{\delta+1} / I_{\delta}$ is a section algebra over $U_{\delta+1} \backslash U_{\delta}$ with fibres $A_{\omega}^{\delta+1}$, for $\omega \in U_{\delta+1} \backslash U_{\delta}$. We now apply Theorem 2.1 .

To prove the final assertion, we need only show that there is a $\delta<\delta_{0}$ such that $L\left(I_{\delta} \rtimes_{\alpha}^{\tau} G\right)=0$, while $L\left(I_{\delta+1} \rtimes_{\alpha}^{\tau} G\right) \neq 0$. Our assertion then follows as any irreducible representation of a continuous field lives on a fibre [3, 10.4.4]. However, from (3) above, it is not hard to show there is a maximal ordinal, $\delta$, with respect to the property that $L\left(I_{\delta} \rtimes_{\alpha}^{\tau} G\right)=0$.

When $G$ and Prim $A$ are second countable and Prim $A$ is almost Hausdorff, then Glimm's theorem implies that Prim $A_{\omega}^{\delta+1}$, which can be identified with $\beta_{\delta+1}^{-1}(\omega)$, is homeomorphic with $G / G_{P}$ for each $P \in \omega$. Define $P^{\delta+1}=P \cap$ $\left(I_{\delta+1} / I_{\delta}\right)$, the primitive ideal of $I_{\delta+1} / I_{\delta}$ associated to the identity coset by this homeomorphism. By [8, Theorem 17], $A_{\omega}^{\delta+1} \rtimes_{\alpha}^{\tau} G$ is strongly Morita equivalent to $\left(A_{\omega}^{\delta+1} / P^{\delta+1}\right) \rtimes_{\alpha}^{\tau} G_{P}$. If in addition, $(G, A)$ is separable, then the former algebra is actually isomorphic to $\left(A_{\omega}^{\delta+1} / P^{\delta+1}\right) \rtimes_{\alpha}^{\tau} G_{P} \otimes \mathscr{K}\left(L^{2}\left(G / G_{P}, \mu\right)\right)$ [9, Theorem 2.13(i)]. Here $\mathscr{K}\left(L^{2}\left(G / G_{P}, \mu\right)\right)$ denotes the compact operators on $L^{2}\left(G / G_{P}, \mu\right)$, where $\mu$ is any quasi-invariant measure on $G / G_{P}$.

If in addition, the action of $G_{P}$ is generalized inner, or more simply, if $A$, hence $A_{\omega}^{\delta+1}$, is GCR, then $A_{\omega}^{\delta+1} / P^{\delta+1} \rtimes_{\alpha}^{\tau} G_{P}$ is isomorphic to

$$
C^{*}\left(G_{P}^{\prime}, \mathbb{C}, \tau^{\prime}\right) \otimes_{\max } A_{\omega}^{\delta+1} / P^{\delta+1}
$$

by [8, Theorem 18]. For the precise definition of $C^{*}\left(G_{P}^{\prime}, \mathbb{C}, \tau^{\prime}\right)$ the reader should consult [8] pages 218 through 220 as well as Theorem 18 . We point out only that $C^{*}\left(G_{P}^{\prime}, \mathbb{C}, \tau^{\prime}\right)$ is a quotient of the group $C^{*}$-algebra of $G_{P}^{\prime}$, where $G_{P}^{\prime}$ is an extension of the circle group by $G_{P} / N_{\tau}$. The representations of $C^{*}\left(G_{P}^{\prime}, \mathcal{C}, \tau^{\prime}\right)$ correspond exactly to $\sigma_{\omega}$-representations of $G_{P} / N_{\tau}$, where $\sigma_{\omega}$ is the (class of the) cocycle corresponding to the extension $G_{P}^{\prime}$.

Fortunately when $A$ is abelian and $\tau$ is trivial, these objects simplify a great deal. $A_{\omega}^{\delta+1}$ is seen to be $C_{0}(G \cdot x)$ and thus, $A_{\omega}^{\delta+1} \rtimes_{\alpha} G$ is isomorphic to 
$C^{*}\left(G, G / G_{x}\right)$. We may apply $[9$, Corollary 8$]$ to conclude that $C^{*}\left(G, G / G_{x}\right)$ is isomorphic to $C^{*}\left(G_{x}\right) \otimes \mathscr{K}\left(L^{2}\left(G / G_{x}, \mu\right)\right)$ in the separable case.

We state two corollaries which illustrate these ideas.

COROLlary 3.3. Suppose that $(G, \Omega)$ is a second countable locally compact transformation group. If $C^{*}(G, \Omega)$ is type $\mathrm{I}$, then $C^{*}(G, \Omega)$ has the structure indicated in Theorem 3.2 with $A_{\omega}^{\delta+1} \rtimes_{\alpha} G$ isomorphic to $C^{*}\left(G_{x}\right) \otimes \mathscr{K}\left(L^{2}\left(G / G_{x}\right)\right)$, where $x \in \omega$.

Proof. If $C^{*}(G, \Omega)$ is type $\mathrm{I}$, then $\Omega / G$ is $T_{0}$ by [11]. The action of $G$ is smooth by Glimm's theorem [10].

The next corollary is due to Takesaki [16] and, when $(G, A)$ is separable and $A$ is GCR, it holds without any amenability assumptions. We include it here primarily as an example of the use of Theorem 3.2.

Corollary 3.4. Suppose that $(A, G, \alpha)$ is a $C^{*}$ dynamical system such that each stability group for the action of $G$ is amenable. In addition, assume that $G$ acts smoothly and the natural maps of $G / G_{P}$ onto $G \cdot P$ are homeomorphisms for each $P$. Then $A \rtimes_{\alpha} G$ is type I if and only if $\left(A^{\delta+1} / P^{\delta+1}\right) \rtimes_{\alpha} G_{P}$ is type I for every $P \in \operatorname{Prim}(A)$. If, in addition, $A$ is type I then $A \rtimes_{\alpha} G$ is type I if and only if every $\sigma_{\omega}$-representation of $G_{P}$ is type $\mathrm{I}$ for every $P \in \operatorname{Prim}(A)$.

Proof. By the previous theorem, we need to show that every representation of $A_{\omega}^{\delta+1} \rtimes_{\alpha} G$ is type I for each $\alpha$. However, $A_{\omega}^{\delta+1} \rtimes_{\alpha} G$ is Morita equivalent to $\left(A_{\omega}^{\delta+1} / P^{\delta+1}\right) \rtimes_{\alpha} G_{P}$ by the result of Greens' mentioned above. The first assertion follows from this.

We note that the fact that Prim $A^{\delta+1}$ is homeomorphic to $G / G_{P}$ implies $P^{\delta+1}$ is maximal in $A^{\delta+1}$. Thus, if $A$ is type $\mathrm{I}, A_{\omega}^{\delta+1} / P^{\delta+1}$ is isomorphic to $\mathscr{K}(\mathscr{H})$, the algera of compact operators on some Hilbert space. Thus, $\left(A^{\delta+1} / P^{\delta+1}\right) \rtimes_{\alpha} G_{P}$ is Morita equivalent to $C^{*}\left(G_{p}^{\prime}, \mathbb{C}, \tau^{\prime}\right)$ when $A$ is type I.

\section{References}

[1] R. C. Busby, 'Double centralizers and extensions of $C^{*}$-algebras,' Trans. Amer. Math. Soc. 132 (1968), 79-99.

[2] R. C. Busby and H. A. Smith, 'Representations of twisted group algebras,' Trans. Amer. Math. Soc. 147 (1970), 503-537.

[3] J. Dixmier, $C^{*}$-algebras, (North-Holland, New York, 1977).

[4] S. Doplicher, D. Kastler, and D. W. Robinson, 'Covariance algebras in field theory and statistical mechanics,' Comm. Math. Phys. 3 (1966), 1-28. 
[5] M. Dupré, 'The classification and structure of $C^{*}$-algebra bundles, Mem. Amer. Math. Soc. 21 (1979), No. 222.

[6] J. M. G. Fell, 'The structure of algebras of operator fields,' Acta Math. 106 (1961), 233-280.

[7] J. M. G. Fell, 'An extension of Mackey's method to Banach *-algebraic bundles,' Mem. Amer. Math. Soc. 90 (1969).

[8] P. Green, 'The local structure of twisted covariance algebras,' Acta Math. 140 (1978), 191-250.

[9] P. Green, 'The structure of imprimitivity algebras,' J. Funct. Anal. 36 (1980), 88-104.

[10] J. Glimm, 'Locally compact transformation groups,' Trans. Amer. Math. Soc. 101 (1961), 124-128.

[11] E. C. Gootman, 'The type of some $C^{*}$ - and $W^{*}$-algebras associated with transformation groups,' Pacific J. Math. 48 (1973), 93-106.

[12] E. C. Gootman and J. Rosenburg, 'The structure of crossed product $C^{*}$-algebras: A proof of the Generalized Effros-Hahn conjecture,' Invent. Math. 52 (1979), 283-298.

[13] R. -Y. Lee, 'On the $C^{*}$-algebras of operator fields,' Indiana Univ. Math. J. 25 (1976), 303-314.

[14] G. K. Pedersen, $C^{*}$-algebras and their automorphism groups, (Academic Press, London, 1979).

[15] M. A. Rieffel, 'Unitary representations of group extensions; an algebraic approach to the theory of Mackey and Blattner,' Studies in Analysis, Adv. Math. Suppl. Studies 4 (1979), 43-81.

[16] M. Takesaki, 'Covariant representations of $C^{*}$-algebras and their locally compact automorphism groups,' Acta Math. 119 (1967), 273-303.

[17] D. P. Williams, 'The topology on the primitive ideal space of transformation group $C^{*}$ algebras and C.C.R. transformation group $C^{*}$-algebras,' Trans. Amer. Math. Soc. 266 (1981), 335-359.

Department of Mathematics

Dartmouth College

Hanover, New Hampshire 03755

U.S.A. 